\title{
Transcultural psychopharmacotherapy
}

\author{
The following are extracts from the Transcultural Psychopharmacotherapy Newsletter, \\ Numbers 4 and $5^{*}$
}

At present millions of people living in different countries and in different cultural settings are using psychotropic drugs prescribed very often on the basis of general instructions. It is highly probable however that the therapeutic efficacy as well as the side effects of such drugs vary significantly from country to country because of cultural, pharmacogenetic, climatic and other factors.

To improve the treatment of mental disorders worldwide, WHO has decided to develop research on transcultural aspects of psychopharmacotherapy. To discuss the most important areas of this field and plan research it was agreed to organise a series of international meetings. WHO also sponsors the Transcultural Psychopharmacotherapy Newsletter, which reports on these meetings, and has published a bibliography of research on transcultural psychopharmacology.

\section{Methodologic difficulties in transethnic| transcultural psychopharmacology}

Transethnic/transcultural psychopharmacology is still a neglected area, although there is increasing interest in this field. This is partly due to difficulties financing projects in centres which are located in various parts of the world. Moreover, methodologic difficulties arise in the design of studies on transethnic/transcultural psychopharmacology.

The diagnostic criteria of DSM-IIIR, the most widely used by investigators in the world, are not all transculturally validated. Therefore samples from various ethnic groups may not be comparable.

The translation and validation of various rating scales has not been achieved in many different languages and cultures.

There may be considerable difficulties matching samples of patients across countries. Patients in developed countries are more likely to be tertiary referrals, with complex medical histories, than patients in developing countries.

The mean age of patients is usually lower in developing countries, compared to patients in developed countries; it is therefore not easy to match for age in samples from different countries. It is worth underlining that there are usually more male in-patients

- Editor: D. Moussaoui (Morocco). Advisory Board: N. Sartorius (Switzerland), L. Prilipko (Switzerland), K. M. Lin (USA), and S. Takahashi (Japan).

in psychiatric wards than females in developing countries, which is the opposite situation of what is usually seen in developed countries.

Defining the ethnicity of patients can be difficult, because it does not always parallel the nationality.

D. MoussaOU, M.D. Casablanca, Morocco

Meeting on transethnic transcultural psychopharmacology: CINP Congress, Nice, France, 28 June-2 July 1992

Following introductory remarks by the President, Dr Kalow, University of Toronto, his colleague Dr Inaba reviewed methodological problems in transcultural psychopharmacology research.

Dr Ishizaki, National Medical Center Clinical Research Laboratory, Tokyo, presented his data of comparing three different oriental populations, Japanese, Korean and Chinese. These three ethnic groups appeared identical as far as phenotyping of debrisoquine/mephenytoin poor metabolizers was concerned. He also presented a patient with depressive disorder who exhibited extraordinarily high plasma concentrations of desmethylimipramine, suggesting possibilities of adverse effect due to poor hydroxylation and extensive desmethylation, that could induce very high plasma concentrations of imipramine and its active metabolite.

Dr Lin, from UCLA Harbor Medical Center, reviewed his project comparing three groups, Caucasians, American born Asians and foreign born Asians. His project involves not only tricyclic antidepressants and haloperidol but benzodiazepines.

In the afternoon session, there were nine presentations. Two papers from Japan and two papers from Canada were studies of pharmacokinetics of tricyclic antidepressants, haloperidol and haloperidol reductase activity.

Dr Yoshida, Los Angeles, brought us up-to-date on crossethnic research on alcohol dehydrogenase (ALDH). He showed the results of gene analysis based on his own data of Oriental type and Caucasian type of human ALDH2 isoenzymes. There are three genotypes of ALDH, and homozygotes (type 1-1) are more likely to suffer from alcoholism.

D. Moussaoui, M.D. S. TAKAHASH, M.D. 